\title{
First-light Science Observations of the Metis Solar Coronagraph
}

S. Fineschi, M. Romoli, V. Andretta, A. Bemporad, G. Capobianco, et al.

S. Fineschi, M. Romoli, V. Andretta, A. Bemporad, G. Capobianco, M. Casti, V. Da Deppo, Y. De Leo, M. Fabi, F. Frassetto, C. Grimani, K. Heerlein, P. Heinzel, G. Jerse, F. Landini, A. Liberatore, E. Magli, G. Naletto, G. Nicolini, M. Pancrazzi, M. G. Pelizzo, P. Romano, C. Sasso, A. Slemer, D. Spadaro, M. Stangalini, T. Straus, R. Susino, L. Teriaca, M. Uslenghi, C.A. Volpicelli, P. Zuppella, "First-light Science Observations of the Metis Solar Coronagraph," Proc. SPIE 11852, International Conference on Space Optics - ICSO 2020, 1185211 (11 June 2021); doi: 10.1117/12.2599221

SPIE Event: International Conference on Space Optics - ICSO 2021, 2021, Online Only 


\section{International Conference on Space Optics-ICSO 2020}

Virtual Conference

30 March-2 April 2021

Edited by Bruno Cugny, Zoran Sodnik, and Nikos Karafolas
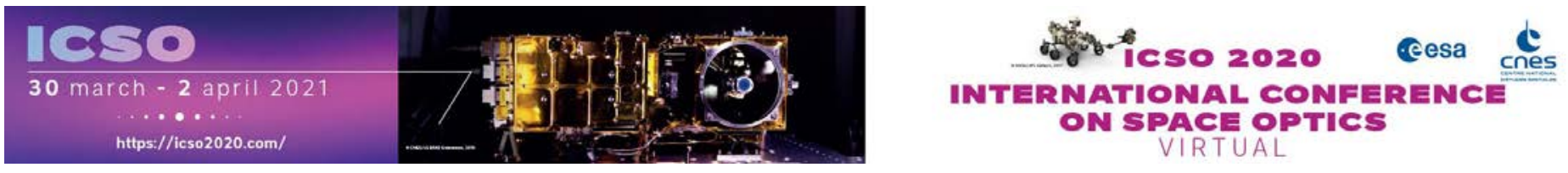

\section{First-light Observations of the Metis Solar Coronagraph}

\section{Cesa isopmeatiant ecnes}

International Conference on Space Optics - ICSO 2020, edited by Bruno Cugny, Zoran Sodnik, Nikos Karafolas, Proc. of SPIE Vol. 11852, 1185211 - (c) 2021 ESA and CNES

CCC code: $0277-786 \mathrm{X} / 21 / \$ 21 \cdot$ doi: $10.1117 / 12.2599221$ 


\title{
First-light Observations of the Metis Solar Coronagraph
}

S. Fineschi ${ }^{\mathrm{a}}$, M. Romoli ${ }^{\mathrm{b}}$, V. Andretta ${ }^{\mathrm{c}}$, A. Bemporad $^{\mathrm{a}}$, G. Capobianco $^{\mathrm{a}}$, M. Casti $^{\mathrm{a}, \mathrm{n}}$, V. Da

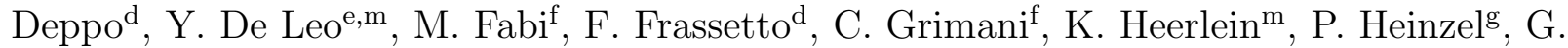
Jerse $^{\mathrm{h}}$, F. Landini ${ }^{\mathrm{a}}$, A. Liberatore ${ }^{\mathrm{a}}$, E. Magli ${ }^{\mathrm{i}}$, G. Naletto ${ }^{\mathrm{j}}$, G. Nicolini ${ }^{\mathrm{a}}$, M. Pancrazzi ${ }^{\mathrm{a}}$, M.G.

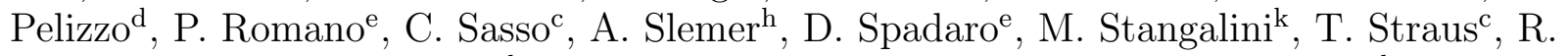
Susino $^{\mathrm{a}}$, L. Teriacal, M. Uslenghi ${ }^{\mathrm{m}}$, C.A. Volpicelli ${ }^{\mathrm{a}}$, and P. Zuppella ${ }^{\mathrm{d}}$

${ }^{a}$ INAF - Osservatorio Astrofisico di Torino, Italy

${ }^{\mathrm{b}}$ Università di Firenze, Italy

${ }^{c}$ INAF - Osservatorio Astronomico di Capodimonte, Napoli, Italy

${ }^{\mathrm{d}} \mathrm{CNR}$ - IFN, Padova, Italy

eINAF - Osservatorio Astrofisico di Catania, Italy

${ }^{\mathrm{f} U n i v e r s i t a ̀ ~ d e g l i ~ S t u d i ~ d i ~ U r b i n o ~ C a r l o ~ B o ~ a n d ~ I N F N, ~ F i r e n z e, ~ I t a l y ~}$

${ }^{\mathrm{g}}$ Academy of Science, Astronomical Institute, Ondrejov, Czech Republic

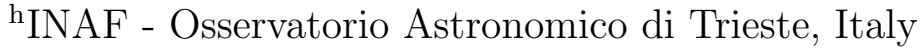

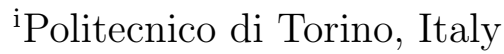

jUniversità di Padova, Italy

${ }^{k}$ Italian Space Agency, Italy

${ }^{1}$ INAF - IASF, Milano, Italy

${ }^{m}$ MPS, Göttingen, Germany

${ }^{\mathrm{n}}$ Catholic University, USA

\begin{abstract}
Metis coronagraph is one of the remote-sensing instruments of the Solar Orbiter mission launched in February 2020. The mission profile will allow for the first time the remote-sensing observation of the Sun from as close as 0.28 Astronomical Units (AU) and from ecliptic latitudes as high as $30^{\circ}$. Metis, in particular, is aimed at the study and the overall characterization of the solar corona and solar wind. This instrument is an innovative inverted-occultation coronagraph that will image the solar corona for the first time simultaneously in two different wavelength band-passes: in the linearly-polarized visible-light (VL), between 580 and $640 \mathrm{~nm}$, and in the ultraviolet (UV) Hi Lyman- $\alpha$ line of hydrogen, at $121.6 \mathrm{~nm}$. The visible channel includes a broad-band polarimeter to observe the linearly polarized component of the K corona. These measurements will allow a complete characterization of the physical parameters, such as density and outflow speed, of the two major plasma components of the corona and the solar wind: electrons (protons) and hydrogen.

After a period of commissioning, by the summer of 2020, Metis has performed the First-light Science Observations during the "Remote-Sensing Check-out Window" (RSCW) that is a telemetry contact period, specifically allocated before entering the operational phase at the end of 2021.

This paper reports the first-light science observations of Metis represented by the UV and polarized VL images of the corona. The resulting first-light maps of the coronal electron distributions are presented. These results are compared to the observations of the visible-light ground-based coronagraph K-Cor and the visibleligh coronagraph LASCO onboard SOHO mission orbiting on the Lagrangian-1 Point and therefore offering a different viewpoint of the solar corona with respect to Solar Orbiter.
\end{abstract}

Keywords: Solar Orbiter, Metis, Sun, coronagraph, solar corona, polarization, electron density.

Further author information: (Send correspondence to Silvano Fineschi)

Silvano Fneschi: E-mail: silvano.fineschi@inaf.it, Telephone: (+39) 0118101919 


\section{INTRODUCTION}

Solar Orbiter, launched from Cape Canaveral, Florida, in February 10 ${ }^{\text {th }} 2020$ at 04:03 UTC aboard a NASAprovided Atlas V 411 launch vehicle, is an ESA-led mission, with strong NASA participation, that will allow for the first time the remote-sensing observation of the Sun from as close as $0.28 \mathrm{AU}$. This spacecraft (S/C) will be progressively more inclined to the ecliptic plane (until an ecliptic latitudes as high as $30^{\circ}$ ) observing, for the first time, the polar regions of the Sun. ${ }^{1}$ On board Solar Orbiter there are 6 remote-sensing instruments and 4 sets of in-situ instruments for a total of 10 different experiments. The goal of the mission is to perform a detailed measurements of the inner heliosphere and solar wind by combining observations from all these instruments. Among the Solar Orbiter remote-sensing instruments, there is the coronagraph Metis*.

Metis instrument is a multi-wavelength externally occulted coronagraph. ${ }^{2,3,4}$ It acquires images of the solar corona in two different wavelengths simultaneously: ultraviolet (UV) and visible light (VL). The UV channel works on the narrow-band Hi Lyman- $\alpha, 121.6 \mathrm{~nm}$, while the VL one works on the broadband 580-640 nm (Fig. 1).

One of the science objectives of Metis coronagraph is to study the Kontinuierlich corona ("K-corona") deriving the electron density in order to characterize the coronal plasma and to determine the origin and acceleration of the fast and slow solar wind. The K-corona, generated by Thomson scattering of the photospheric light from free coronal electrons, is linearly polarized (e.g., Refs. 5, 6) In particular, it is well known that the K-corona brightness is directly proportional to the electron column density along the line-of-sight. Then, by measuring the brightness and adopting proper geometrical assumptions, it is possible to estimate the electron density. ${ }^{5}$ These observations will contain not only the K-corona but also a component due to the scattering of photospheric light from dust as well as a stray light component. In order to eliminate these components, Metis will measure the polarized brightness (pB) - defined as the difference between polarization component tangential to the solar limb and that parallel to the solar radius. Along the optical path of the visible channel, a polarimeter assembly with the presence of an electro-optically modulating Liquid Crystal Variable Retarder (LCVR) would measure the linear polarization brightness $(\mathrm{pB})$ of the K-corona (through the acquisition of 4 images with different angle of polarizations). ${ }^{7,8,9}$ From the $\mathrm{pB}$ the coronal electron density can be derived.

Section 2 describes in more details the Metis optical design and characteristics. Section 3, reports the firstlight images of the visible-light polarized K-corona and of the UV Hi Lyman- $\alpha$ line-emission acquired by Metis on May $15^{\text {th }}, 2020$. The same section shows the composite images of Metis polarized $\mathrm{pB}$ and UV observations with with the $\mathrm{pB}$ observations from the coronagraphs LASCO onboard SOHO and the ground-based, High Altitude Observatory (HAO) K-Cor. Finally, Section 4 shows the cross-calibration of Metis with LASCO and K-Cor. This cross-calibration is then used to derive the coronal electron density.

\section{METIS CORONAGRAPH}

Due to the Solar Orbiter wide range of distances from the Sun, the on-board instruments need a particular design to be able to sustain the high thermal flux at the perihelion. For this reason, the Metis occultation scheme is based on an "inverted" external-occulter (IEO) to reduce the high thermal load (temperatures up to $400{ }^{\circ} \mathrm{C}$ ) on the instrument when the spacecraft is nearest to the Sun. In a classical externally-occulted coronagraph, the annular disk-light rejecting mirror is much larger than the circular one used in the inverted-occultation scheme (M0 in Fig. 1). This reduces by two orders of magnitude the thermal load on the rejection mirror. ${ }^{3}$ The coronal light is collected by an on-axis aplanatic Gregorian telescope. The suppression of the diffracted light off the edges of the IEO and M0 is achieved, respectively, with an internal occulter (IO) and a Lyot trap (LS). ${ }^{4}$

The primary (M1) and secondary (M2) telescope (see Fig. 1) have a $\mathrm{Al} / \mathrm{MgF}_{2}$ coating to enhanced reflectivity at HI Lyman- $\alpha 121.6 \mathrm{~nm}$ and with high reflectivity also in the visible wavelength range $580-640 \mathrm{~nm} .{ }^{2}$ The coronal light is then splitted by an UV interference filter that works by selecting the $121.6 \mathrm{~nm}$ UV band in transmission and reflecting the VL into the polarimeter where the broadband filter selects the VL bandpass 580-640 nm. ${ }^{7,10,11}$ The inset in Figure 1 shows the detail of the Metis polarimeter.

The platescale of the UV and VL detectors are $20 \mathrm{arcsec} /$ pixel and $10 \mathrm{arcsec} /$ pixel respectively. All these, and others, optical characteristics are summarized in Tab. 1. More detailed information on the Metis instrument can be found in Ref. 3, 4 .

\footnotetext{
*In ancient Greek mythology, Metis was the first wife of Zeus and symbol of wisdom and deep thought.
} 


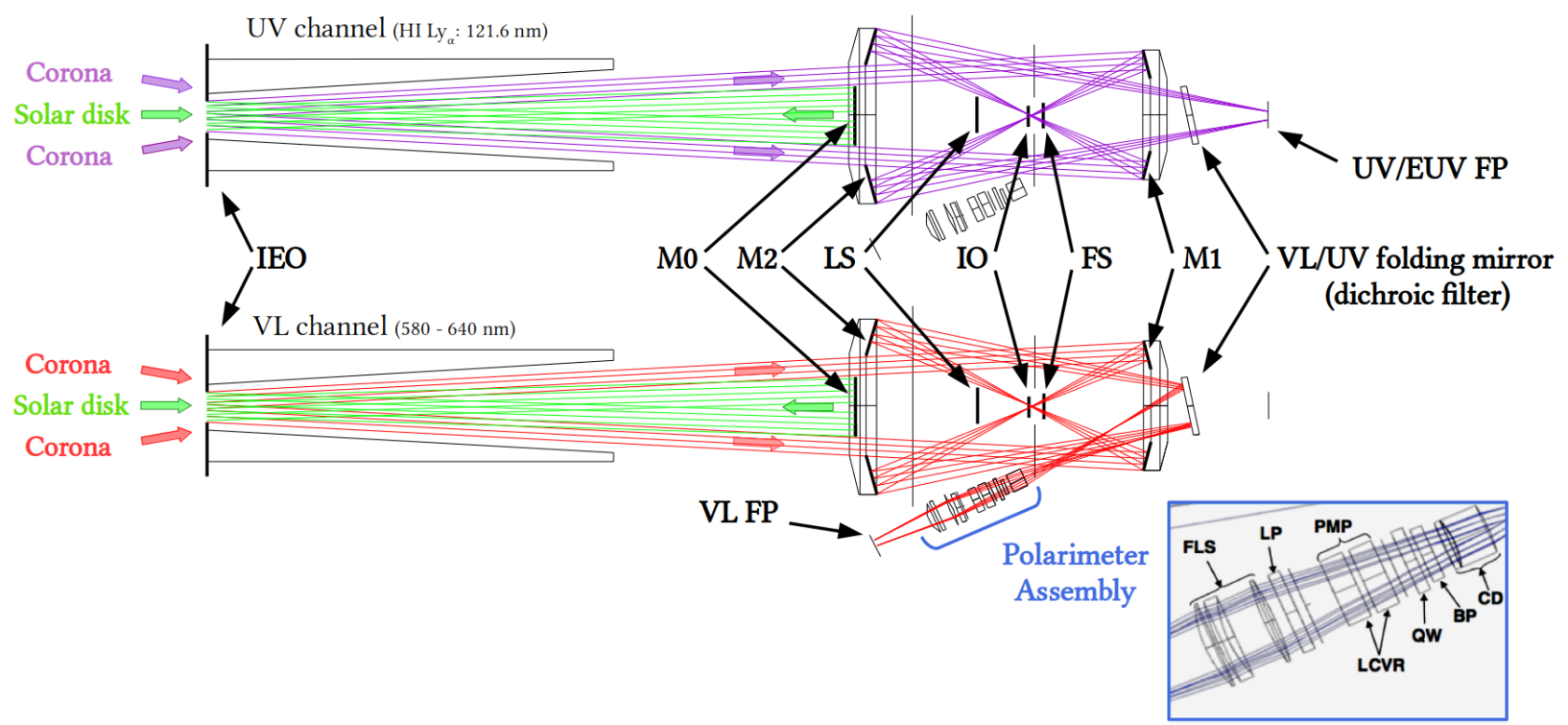

Figure 1: Metis ray trace for the UV (top) and VL (bottom) channels. The inset on the bottom, right-hand side shows the polarimeter with its optical components: a collimating doublet (CD), the VL bandpass filter (BP), focus lens system (FLS), a quarter-wave (QW) plate retarder, a polarisation modulation package (PMP) comprising two liquid crystals variable retarders (LCVRs). ${ }^{7,9}$

Figure 2 (left) shows the Metis' field-of-view (FoV) in units of heliocentric heights when Solar Orbiter's distance from the Sun is $0.25 \mathrm{AU}$. The table on the right in the same figure shows the changing FoV for different Sun-Solar Orbiter distances.

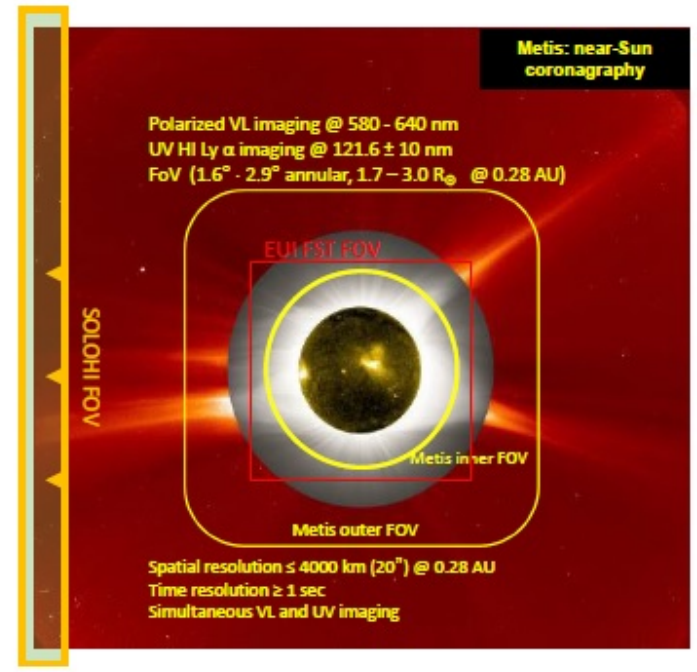

\begin{tabular}{|c|c|c|c|}
\hline $\begin{array}{c}\text { SolO-Sun } \\
\text { Distance } \\
(\mathrm{AU})\end{array}$ & Min FOV & Max FOV & Corner FOV \\
\hline 0.28 & $1.7 \mathbf{R}_{\odot}$ & $3.0 \mathbf{R}_{\odot}$ & $3.9 \mathrm{R}_{\odot}$ \\
\hline $\mathbf{0 . 3}$ & $\mathbf{1 . 7 5 \mathbf { R } _ { \odot }}$ & $\mathbf{3 . 3} \mathbf{R}_{\odot}$ & $4.0 \mathbf{R}_{\odot}$ \\
\hline $\mathbf{0 . 4}$ & $\mathbf{2 . 2 \mathbf { R } _ { \odot }}$ & $\mathbf{4 . 4 \mathbf { R } _ { \odot }}$ & $6.2 \mathbf{R}_{\odot}$ \\
\hline 0.5 & $\mathbf{2 . 8 \mathbf { R } _ { \odot }}$ & $\mathbf{5 . 4 \mathbf { R } _ { \odot }}$ & $7.7 \mathbf{R}_{\odot}$ \\
\hline 0.8 & $\mathbf{4 . 3 \mathbf { R } _ { \odot }}$ & $\mathbf{1 2 \mathbf { R } _ { \odot }}$ & $17 \mathbf{R}_{\odot}$ \\
\hline
\end{tabular}

Figure 2: Left: Field-of-View of Metis in heliocentric height at 0.25 AU. Right: Field-of-View as corresponding to different Solar Orbiter to Sun distances in AU units. 
Table 1: Main optical performances of Metis instrument. ${ }^{4,3}$

\begin{tabular}{|ll|}
\hline Spectral range & \\
\hline Multi-wavelength & VL: $580-640 \mathrm{~nm}$ \\
& UV: Hi Lyman- $\alpha, 121.6 \pm 7.5 \mathrm{~nm}$ (FWHM) \\
\hline Telescope & (Inverted) Externally occulted, on-axis aplanatic Gregorian \\
\hline Type & $1309 \mathrm{~mm}$ \\
Overall length & VL: $200 \mathrm{~mm}$ \\
Effective focal length & $\mathrm{UV}: 300 \mathrm{~mm}$ \\
& \\
\hline Focal Plane & $\mathrm{VL}: 10 \mathrm{arcsec} /$ pixel \\
& $\mathrm{UV}: 20 \mathrm{arcsec} /$ pixel \\
Plate scale & $\mathrm{VL}: 20.7 \mathrm{~mm}(2048 \times 2048)$ with $10 \mu \mathrm{m}$ pixel size \\
& $\mathrm{UV}: 30.7 \mathrm{~mm}(1024 \times 1024)$ with $30 \mu \mathrm{m}$ equivalent pixel size \\
Image size & $1.6^{\circ}-2.9^{\circ}$ annular, off-limb corona \\
& $1.7-3.1 R_{\odot} @ 0.28 \mathrm{AU}$ \\
Field of view (FoV) & $3.0-5.5 R_{\odot} @ 0.5 \mathrm{AU}$ \\
& $\mathrm{VL} B_{\text {stray }} / B_{\odot}<10^{-9}$ \\
& $\mathrm{UV} B_{\text {stray }} / B_{\odot}<10^{-5}$ \\
Average instrumental stray light & $\leq 10^{-2}$ \\
(stray light to solar-disk irradiance ratio) & \\
Broad-band linear polarization sensitivity & \\
\hline
\end{tabular}

\section{METIS FIRST-LIGHT OBSERVATIONS}

Metis acquired the first-light images on May $15^{\text {th }}, 2020$ starting at 11:20 UT, just after the commissioning activity relative to the centerting the internal-occulter (Activity IT-6B1, cfr. Romoli et al., this volume)

Figure 3 shows the position of Solar Orbiter on that day relative to Earth and the Sun. The orbital plot is in geocentric solar ecliptic coordinates, so Earth is at $[0,0]$, the Sun is at $[1,0]$. The plot is the projection of the orbit on the ecliptic plane ${ }^{\dagger}$. The Solar Orbiter's distance from the Sun was 0.64 AU. The angle Solar Orbiter-Sun-Earth was $15^{\circ}$. This allowed for coordinated observations with the ground-based coronagraph K-Cor of the High Altitude Observatory (HAO), ${ }^{12}$ and the LASCO coronagraph onboard SOHO. ${ }^{13}$

\subsection{Visible-light Polarized-Brightness and UV Images}

Figure 4 shows the Metis first-light images in polarized visible-light, within 580-640 nm wavelength bandpass (left, shown in red) and Hi Lyman- $\alpha$ (right, shown in blue) acquired on May $15^{\text {th }}, 2020$.

The images clearly show the two bright equatorial streamers and fainter polar regions, which are characteristic of the solar corona during times of minimal magnetic activity. The ultraviolet image (shown in blue) records emission from neutral hydrogen atoms in the corona. This is the first UV image of the extended solar corona ever obtained. When Metis acquired these images, Solar Orbiter was at a distance of 0.64 AU from the Sun and the instrument field of view covered heliocentric heights $\left(R_{\odot}\right)$ from $3.8 R_{\odot}$ to $7.0 R_{\odot}$ (cfr. Table in Fig. 2).

\footnotetext{
${ }^{\dagger}$ https://issues.cosmos.esa.int/solarorbiterwiki/display/SOSP/LTP01+May+2020-June+2020
} 

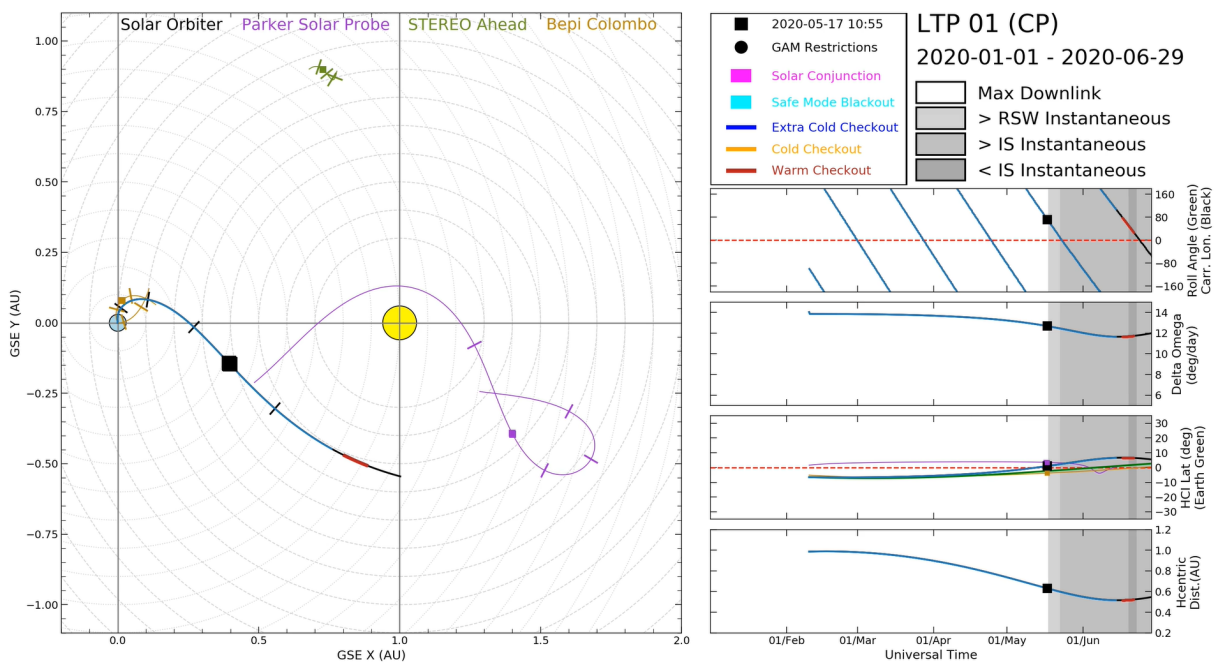

Figure 3: Orbital plot of the Solar Orbiter in geocentric solar ecliptic coordinates. Earth is at $[0,0]$, the Sun is at $[1,0]$. The plot is the projection of the orbit on the ecliptic plane. On May $15^{\text {th }}, 2020$, Solar Orbiter was at a distance of $0.64 \mathrm{AU}$ (black square). The angle Solar Orbiter-Sun-Earth was $15^{\circ}$.

\section{0/05/15 11:39 UT - 11:41 UT}
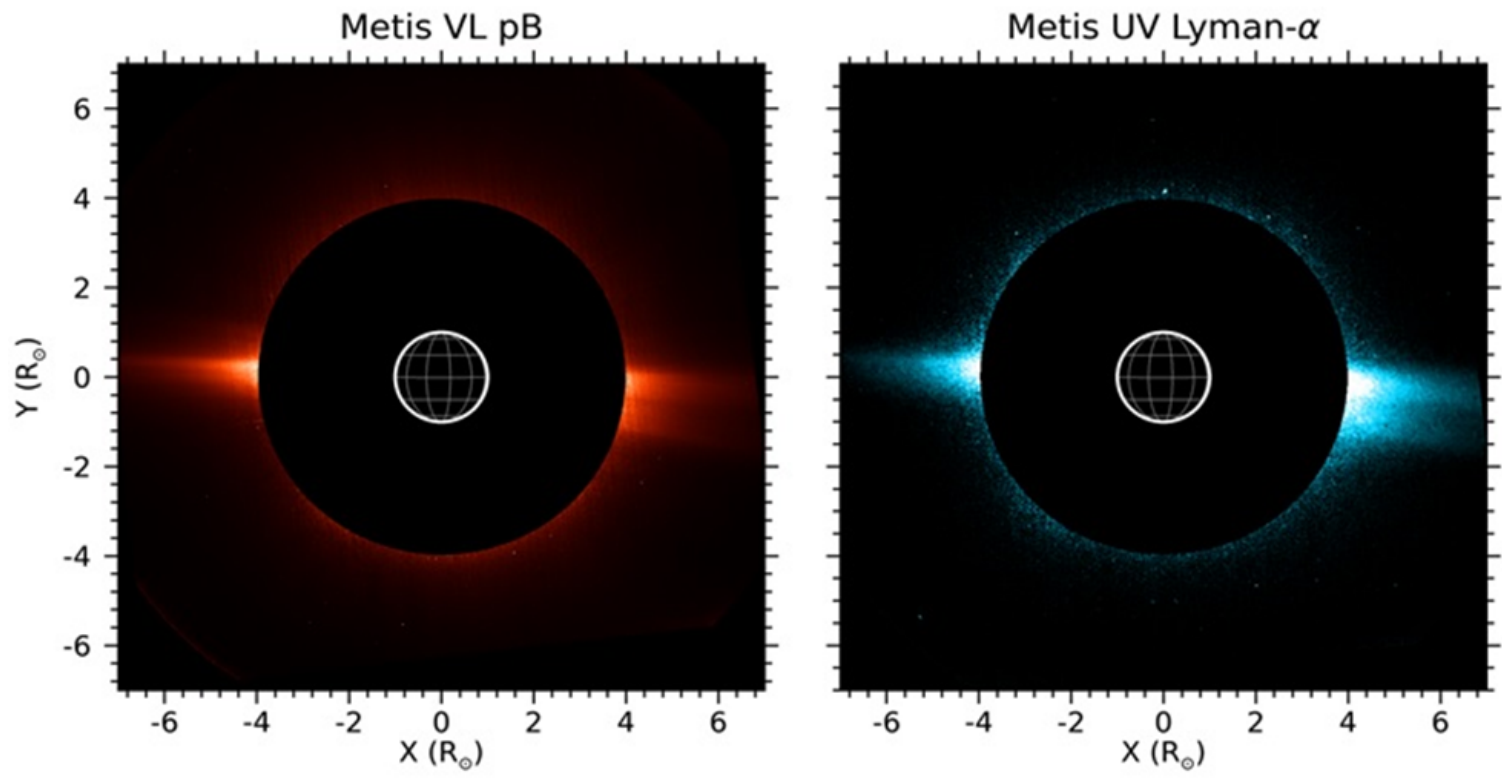

Figure 4: Metis first-light images, from a vantage point of $0.64 \mathrm{AU}$, acquired on May $15^{\text {th }}, 2020$ at $11.39 .25 \mathrm{UT}$, within a field-of-view ranging from $3.8 R_{\odot}$ and $7 R_{\odot}$. Left: VL pB. Right: Hi Lyman- $\alpha$ at $121.6 \mathrm{~nm}$.

On May $15^{\text {th }}, 2020$, two VL pB sequences were acquired with detector integration time equal to $15 \mathrm{~s}$ and $30 \mathrm{~s}$, respectively, and two sets of $6 \mathrm{UV}$ images each with integration time of $8 \mathrm{~s}$ and $16 \mathrm{~s}$, respectively. Both VL and UV detectors had a $1 \times 1$ pixel binning, corresponding to an image scale on the plane of the sky of about $4300 \mathrm{~km}$ and $8600 \mathrm{~km}$, respectively. Figure 4 shows on the left the VL pB obtained with integration time of $30 \mathrm{~s}$. The corresponding UV Hi Lyman- $\alpha$ corona image acquired with integration time of $16 \mathrm{~s}$ is shown on the right. 
The set of images were processed and calibrated by applying bias and dark subtraction, flat-field and vignetting correction, exposure-time normalization, and radiometric calibration. The bias and dark images for both detectors were acquired in flight, whereas flat-field and vignetting images as well as the radiometric parameters, used to convert the image values from digital number to the physical units, were measured on ground during laboratory calibrations ${ }^{4}$ carried out at the INAF Optical Payload Systems - OPSys - facility in ALTEC. ${ }^{14}$ The VL pB images were obtained from sets of four polarimetric VL images combined with the demodulation matrix obtained by inverting the polarimeter's Muller matrix derived from on-ground calibrations. ${ }^{10,11}$

The ultraviolet image of the emission from neutral hydrogen atoms in Figure 4 is the first Hi Lyman- $\alpha$ image of the outer solar corona ever obtained, in this case extending up to $6.0 R_{\odot}$. The brightness distribution in the field-of-view is very similar to that of the visible light polarized brightness with the two bright equatorial streamers and fainter polar regions. The streamers appear significantly bright all the way to the upper limit of the field-of-view, showing the presence of emitting neutral hydrogen at large distances from the Sun.

\subsection{Coordinated observations with LASCO and K-Cor}

During the Metis observations on May $15^{\text {th }}$, 2020, the LASCO-C2 coronagraph onboard SOHO and the HAO, ground-based coronagraph K-Cor were also acquiring pB images. The LASCO-C3 coronagraph acquired pB images on May $14^{\text {th }}$ and $16^{\text {th }}, 2020$.

Figure 5 shows on the left-hand side the combined coronal images of Metis VL (green), LASCO-C2 (red) and K-Cor (deep blue) on the left. The same is shown on the right-hand side, but with the coronal image of Metis UV (light blue). K-Cor field-of-view (FoV) covers heliocentric heights from $1.01 R_{\odot}$ to $3 R_{\odot} \cdot{ }^{12}$ LASCO-C2 field-of-view (FoV) covers heliocentric heights from $1.5 R_{\odot}$ to $6 R_{\odot}{ }^{13}$ Metis field-of-view (FoV) at $0.64 \mathrm{AU}$ covers heliocentric heights from $3.8 R_{\odot}$ to $7.0 R_{\odot}$. Thus, on May $15^{\text {th }}, 2020$, the FoVs of all three coronagraphs guaranteed a good overlap in their respective coverages of the corona. The images from these coronagraphs allowed the imaging of the corona from $1.01 R_{\odot}$ to $6.5 R_{\odot}$ in VL and - for the first time - the comparison with the UV coronal line-emission Hi Lyman- $\alpha$ from $3.8 R_{\odot}$ to about $7 R_{\odot}$.
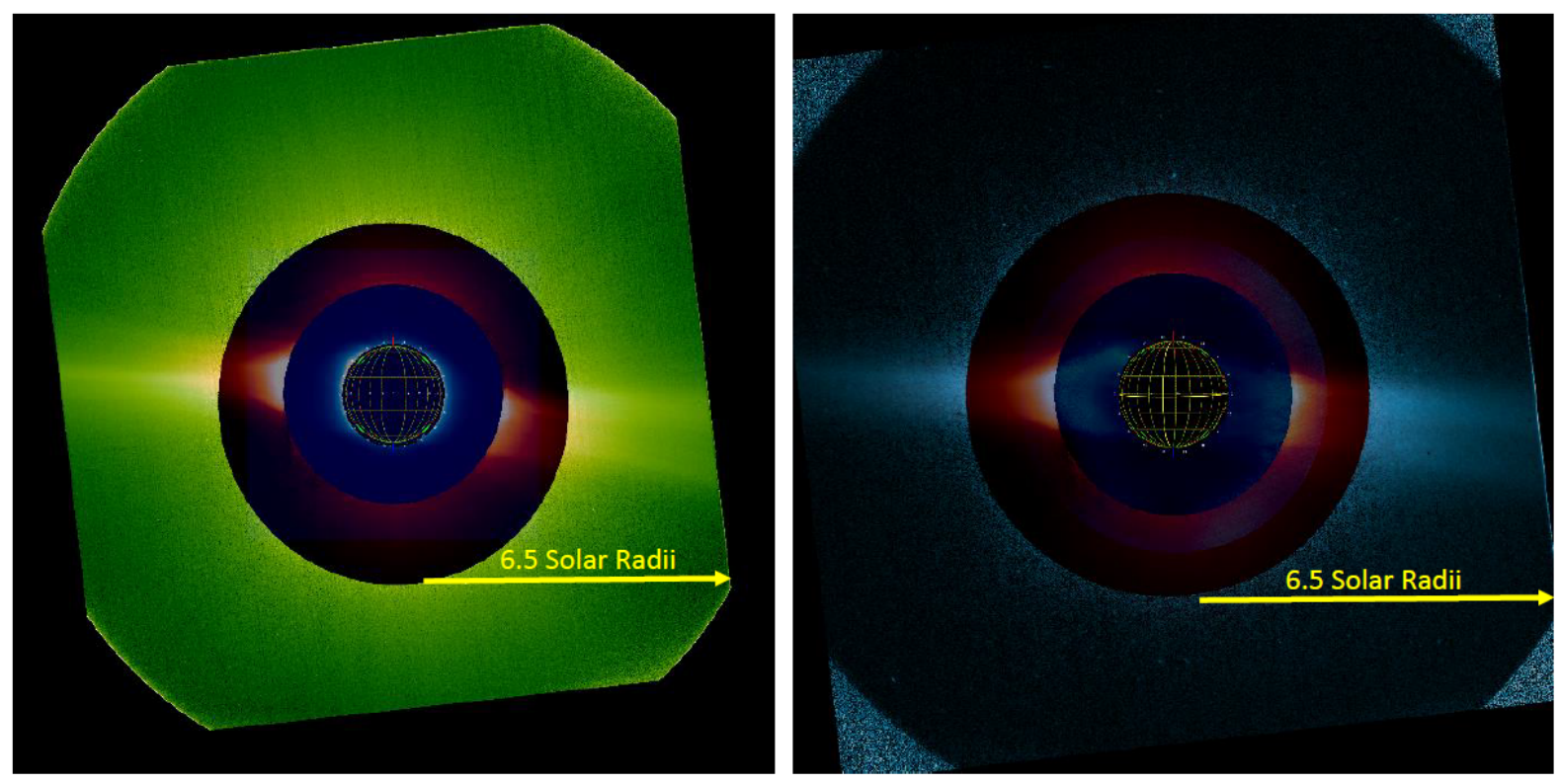

Figure 5: Left: Combined coronal images of Metis VL (green), LASCO-C2 (red) and K-Cor (deep blue). Right: Same as in the image on the left, but with the coronal image of Metis UV (light blue). K-Cor field-of-view (FoV) covers heliocentric heights from $1.01 R_{\odot}$ to $3 R_{\odot} \cdot{ }^{12}$ LASCO-C2 field-of-view (FoV) covers heliocentric heights from $1.5 R_{\odot}$ to $6 R_{\odot} \cdot{ }^{13}$ Metis field-of-view (FoV) at $0.64 \mathrm{AU}$ covers heliocentric heights from $3.8 R_{\odot}$ to $7.0 R_{\odot}$. 
Images of the coronal VL pB broadband emission acquired by LASCO-C3 (FoV: $\left.3.7 R_{\odot}-30 R_{\odot}\right)$ allowed a similar comparison at heliocentric heights up of $30 R_{\odot}$, as shown on the image on the right-hand side in Figure 6

The composite image on the left-hand side in Figure 6 shows the entire corona obtained by combining images acquired by Metis in polarized light (red), by K-Cor, by the AIA instrument ${ }^{15}$ (19.3 nm, solar disk) on board the SDO probe, ${ }^{16}$ and the reconstruction of the global structure of the magnetic field obtained starting from extrapolations of the photospheric magnetic field. $\ddagger$ In the quiet phases of the solar activity cycle, the Sun’s global magnetic field confines the plasma mainly to the equatorial belt, where the field lines are closed, thus giving rise to bright structures.
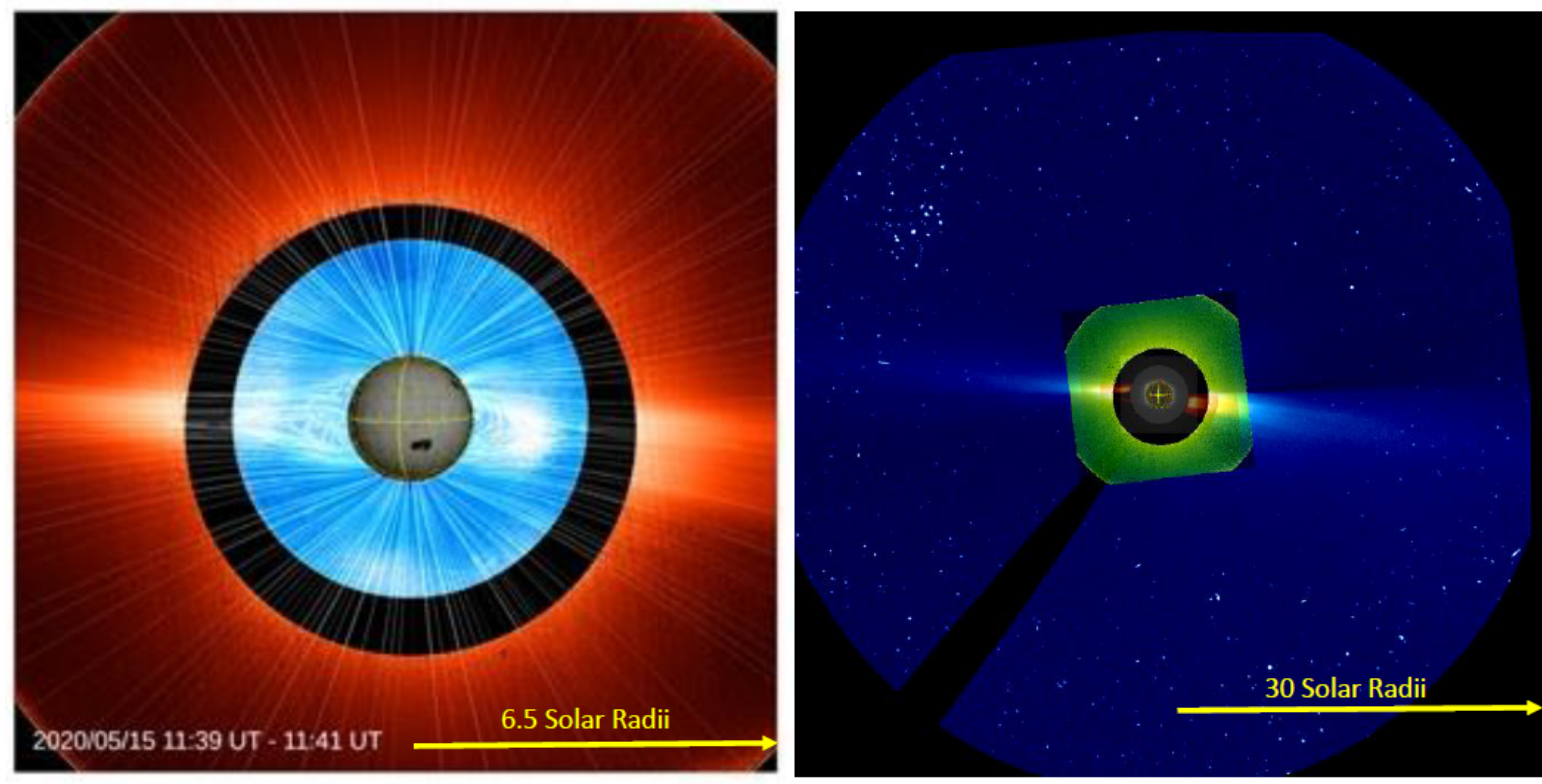

Figure 6: Left: Composite image with Metis VL pB (red), K-Cor VL pB (blue) and AIA instrument ${ }^{15}(19.3 \mathrm{~nm}$, solar disk) on board SDO. ${ }^{16}$ Superposed is the reconstruction of the global structure of the magnetic field obtained starting from extrapolations of the photospheric magnetic field. ${ }^{\ddagger}$. Right: Combined coronal images of Metis VL pB (green), LASCO-C3 pB (blue) and LASCO-C2 pB (red).

The composite image of the coronal VL pB clearly shows the characteristic configuration of the solar corona during the minimum phase of magnetic activity, when the global scale solar magnetic field is predominantly dipolar and confines the plasma mostly near the equatorial belt, giving rise to two bright streamers. The polar regions, where the magnetic field lines are open, exhibit a fainter brightness due the plasma outflow in the solar wind. The classic solar minimum configuration of the solar corona observed with Metis on May $15^{\text {th }}, 2020$, shaped by a predominantly dipolar magnetic field and characterized by an equatorial streamer belt, closely resembles the simple and persistent dipolar configuration observed during 1996, that is, one full magnetic cycle earlier when the streamer belt and the coronal current sheet were only slightly warped around the equatorial plane. At the limb, on the plane of the sky, this is viewed as two almost equatorial streamers stretching out to form the coronal and, further out, the heliospheric current sheet. The 1996 solar minimum corona, studied extensively in the first year of the $\mathrm{SOHO}$ coronagraphs observations, is described, for example, in Ref. 17 by combining the observations of the three LASCO coronagraphs, thus ensuring a continuously coverage of the off-limb corona from $1.1 R_{\odot}$ to $32 R_{\odot}$.

\footnotetext{
${ }^{\ddagger}$ Courtesy of Predictive Science Inc. https://www.predsci.com/portal/home.php
} 


\section{CORONAL ELECTRON DENSITY DERIVED FROM POLARIZED BRIGHTNESS}

The line-of-sight of Metis onboard Solar Orbiter was very closely aligned (about $15^{\circ}$, cfr. Fig. 3) to those of the ground-based K-Cor and of LASCO onboard SOHO on the Lagrangian-1 Point. This, together with the overlap of the Metis' FoV with those of K-Cor and LASCO-C2, -C3, allowed the first in-flight, radiometric cross-calibration of the Metis' VL polarimetric channel.

\subsection{Polarized Brightness cross calibration with K-Cor and LASCO}

The radiometrically calibrated $\mathrm{pB}$ maps derived from the Metis data have been compared with those obtained from K-Cor and LASCO-C2, -C3.

The West and East streamers, having the higher signal, were selected for this calibration. Figure 7 shows the VL pB profiles, in units of mean solar disk brightness $\left(B_{\odot}\right)$, of Metis, K-Cor and LASCO-C3, -C3 as a function of heliocentric heights along the axes of the West and East streamers (left- and right-hand side plots, respectively).

At the heliocetric heights of ovelap, the $\mathrm{pB}$ values from the four coronagraphs compare relatively well, considering that for the reduction of the LASCO data was used the standard LASCO calibration provided in the Solarsoft package ${ }^{\S}$ instead of the most recent radiometric calibration obtained from the reduction of 24 years of LASCO observations. ${ }^{18,19}$ There are no LASCO-C3 observations of the coronal pB on May $15^{\text {th }}$, 2020, but only on May $14^{\text {th }}$ and $16^{\text {th }}$. During the minimum of the solar cycle, the steady-state corona is not expected to vary considerably over the time period of two to three day, and at the spatial scale at which these observations were performed (i.e., tens of arcsec). Also, the projection effects due to the solar rotations have been neglected, in first approximation, over that time interval. The differences between the LASCO-C2 pB values and those of LASCO-C3 may be due more likely to the use of not the most updated versions of their calibration, rather than to the difference in the observing days. Indeed, on the West streamer, Metis $\mathrm{pB}$ profile on May $15^{\text {th }}$ matches better that of LASCO-C2, also on May $15^{\text {th }}$, whereas on the East streamer it matches better that of LASCO-C3, on May $14^{\text {th }}$ and $16^{\text {th }}$.

The K-Cor data above $2 R_{\odot}$ are affected by considerable noise due to the sky-brightness and have not been considered. Nevertheless, the gradient of the pB profiles of K-Cor matches quite well that of Metis.
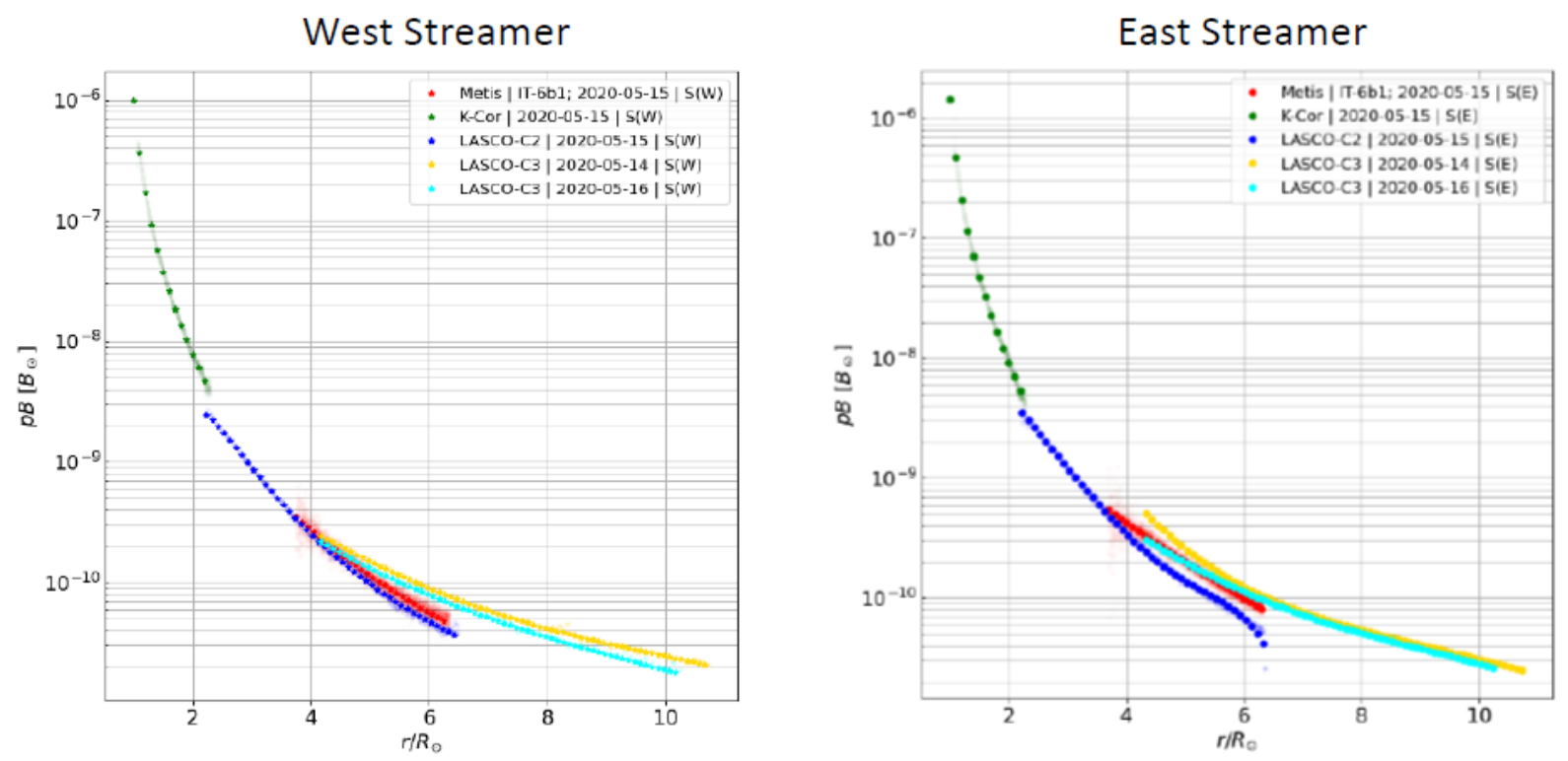

Figure 7: Comparison among Metis, K-Cor and LASCO-C3, -C3 of their VL pB profiles as a function of heliocentric heights along the axes of the West and East streamers (left- and right-hand side, respectively).

\footnotetext{
${ }^{\S}$ https://sohowww.nascom.nasa.gov/solarsoft/.
} 


\subsection{Coronal Electron Density}

The pB maps of all four coronagraphs were inverted to derive the coronal electron densities by assuming a simple spherically symmetric corona, following the classical approach described in Ref. 5.

As expected, the electron density profiles compare well among themselves, reflecting some of the differences already present in the $\mathrm{pB}$ profiles. Also in this case, for instance, on the West streamer, Metis electron density profile on May $15^{\text {th }}$ matches better that of LASCO-C2, also on May $15^{\text {th }}$, whereas on the East streamer it matches better that of LASCO-C3, on May $14^{\text {th }}$.

Figure 8 shows the resulting coronal electron densities profiles (in units of $\left[\mathrm{cm}^{-3}\right]$ ) as a a function of heliocentric heights along the axes of the West and East streamers (left- and right-hand side plots, respectively).
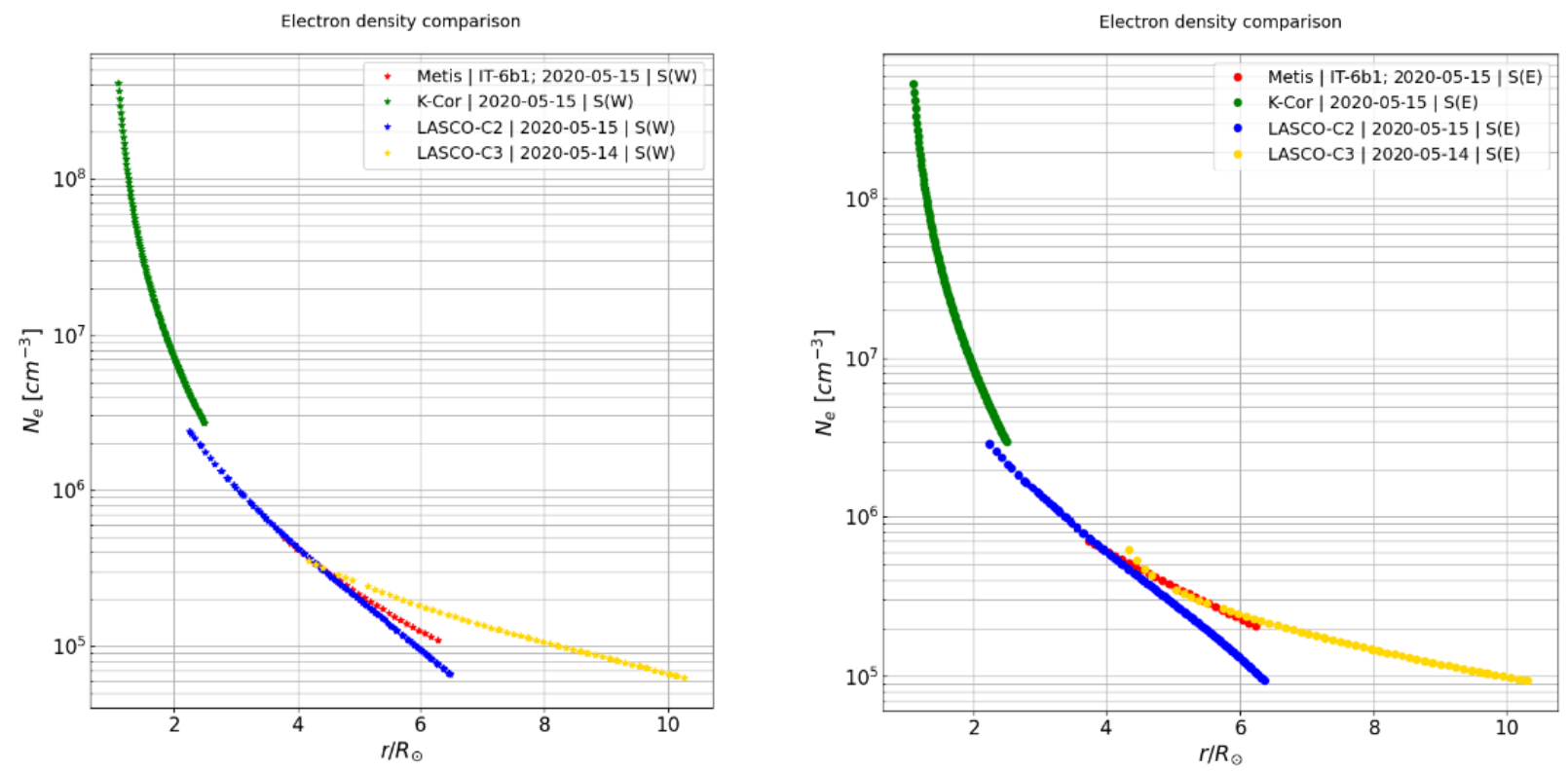

Figure 8: Coronal Electron density profiles as a a function of heliocentric heights along the axes of the West and East streamers (left- and right-hand side plots, respectively). The electron density maps of all four coronagraphs were derived by inverting the $\mathrm{pB}$ with the simplifying assumption of a spherically symmetric corona. ${ }^{5}$

These results, while very preliminary, nonetheless they show that the ground calibration of the Metis VL channel is still valid, in first approximation, also in flight. Additional and regular cross-calibrations will take place during the nominal mission, not only of the VL channel, but also of the UV one. In the meanwhile, the VL pB measurements by Metis can already be used to derive the electron density of the corona from the different observation viewpoints made possible by Solar Orbiter as it progresses along its orbit.

The electron density is the cornerstone physical parameter of the corona. For instance, this quantity is needed to derive - together with the UV measurements of the Hi Lyman- $\alpha$ line-emission - the solar wind speed in the outer corona through to the Doppler-dimming diagnostics. ${ }^{20,21}$

The VL pB and UV first-light images of Metis reported in this paper have been recently used to derive the velocity maps of the expanding solar corona, about $\pm 10^{\circ}$ wide, centered on the extension of the quiet equatorial streamers axis present at the West and East limb. ${ }^{22}$ The electron densities derived for those streamers and used for this Doppler dimming diagnostics are the ones presented in this paper. 


\section{CONCLUSION}

On May $15^{\text {th }} 2020$, Metis onboard Solar Orbiter acquired its first-light images of the visible-light (VL) polarized brightness (pB) of the K-corona and of the ultraviolet (UV) HI Lyman- $\alpha$ line-emission from the coronal hydrogen. This was the first time that the same instrument observed the corona simultaneously in two different wavelength band-passes: in the linearly-polarized VL, between 580 and $640 \mathrm{~nm}$, and in the UV Hi Lyman- $\alpha$ line of hydrogen, $121.5 \mathrm{~nm}$. Solar Orbiter was at 0.65 AU from the Sun. The line-of-sight of Solar Orbiter was very closely aligned to those of the ground-based K-Cor and of LASCO onboard SOHO on the Lagrangian-1 Point. This, together with the overlap of the Metis' FoV with those of K-Cor and LASCO-C2, -C3, allowed the preliminary in-flight radiometric cross-calibration of the Metis' VL polarimetric channel. The comparison of the Metis $\mathrm{pB}$ maps to those of K-Cor and LASCO indicates that the on-ground calibration of the Metis VL polarimetric channel is reliable. The electron densities derived from these VL pB observations will be used - together with the UV measurements - to derive velocity maps of the expanding solar corona through the Doppler dimming diagnostics.

\section{ACKNOWLEDGMENTS}

This paper has been possible thanks to the whole Metis team and the contributions of the many listed authors. Among the others, a particular acknowledgment is due to E. Antonucci, who led this Project as Principal Investigator until the delivery of the instrument to ESA in September 2017, when M. Romoli took over this role.

This work has been supported by the Italian Space Agency - ASI. Metis team thanks the former PI, Ester Antonucci, for leading the development of Metis until the final delivery to ESA. The industrial contractor for the Metis project has been a temporary consortium between OHB Italia (for opto-mechanical design, electronics and the software) and Thales Alenia Space Italia (for telescope thermal and structural design and realization, application software, instrument integration, alignment and test). The primary and secondary mirrors were provided as Czech contribution to Metis; the mirror hardware development and manufacturing was possible thanks to the Czech PRODEX Programme. The authors thank also ALTEC Company for providing logistic and technical support.

\section{REFERENCES}

[1] D. Müller, O. C. St. Cyr, I. Zouganelis, H. R. Gilbert, R. Marsden, T. Nieves-Chinchilla, E. Antonucci, F. Auchère, D. Berghmans, T. S. Horbury, and et al., "The solar orbiter mission," Astronomy \& Astrophysics 642, p. A1, Sep 2020.

[2] S. Fineschi, E. Antonucci, D. Gardiol, V. da Deppo, G. Naletto, M. Romoli, A. Cacciani, and M. Malvezzi, "Extended UV corona imaging from the Solar Orbiter: the Ultraviolet and Visible-light Coronagraph (UVC)," in Solar encounter. Proceedings of the First Solar Orbiter Workshop, B. Battrick, H. SawayaLacoste, E. Marsch, V. Martinez Pillet, B. Fleck, and R. Marsden, eds., ESA Special Publication 493, pp. 217-222, Sept. 2001.

[3] S. Fineschi, G. Naletto, M. Romoli, and et al., "Optical design of the multi-wavelength imaging coronagraph metis for the solar orbiter mission," Experimental Astronomy 49, May 2020.

[4] E. Antonucci, M. Romoli, V. Andretta, S. Fineschi, P. Heinzel, J. D. Moses, G. Naletto, G. Nicolini, D. Spadaro, L. Teriaca, and et al., "Metis: the solar orbiter visible light and ultraviolet coronal imager," Astronomy 83 Astrophysics 642, p. A10, Sep 2020.

[5] H. C. Van De Hulst, "The electron density of the solar corona," B.A.N. 11, p. 135, Feb. 1950.

[6] N. E. Raouafi, "Coronal Polarization," in Solar Polarization 6, J. R. Kuhn, D. M. Harrington, H. Lin, S. V. Berdyugina, J. Trujillo-Bueno, S. L. Keil, and T. Rimmele, eds., Astronomical Society of the Pacific Conference Series 437, p. 99, Apr. 2011.

[7] S. Fineschi, L. Zangrilli, G. Rossi, L. Gori, M. Romoli, G. Corti, G. Capobianco, E. Antonucci, and E. Pace, "KPol: liquid crystal polarimeter for K-corona observations from the SCORE coronagraph," in Solar Physics and Space Weather Instrumentation, S. Fineschi and R. A. Viereck, eds., Society of Photo-Optical Instrumentation Engineers (SPIE) Conference Series 5901, pp. 389-399, Aug. 2005. 
[8] L. Zangrilli, S. Fineschi, and G. Capobianco, "Calibration of the EKPol K-corona imaging polarimeter," in Solar Physics and Space Weather Instrumentation III, S. Fineschi and J. A. Fennelly, eds., Society of Photo-Optical Instrumentation Engineers (SPIE) Conference Series 7438, p. 74380W, Aug. 2009.

[9] A. Alvarez-Herrero, N. Uribe-Patarroyo, P. G. Parejo, J. Vargas, R. L. Heredero, R. Restrepo, V. MartínezPillet, J. C. del Toro Iniesta, A. López, S. Fineschi, G. Capobianco, M. Georges, M. López, G. Boer, and I. Manolis, "Imaging polarimeters based on liquid crystal variable retarders: an emergent technology for space instrumentation," in Polarization Science and Remote Sensing V, J. A. Shaw and J. S. Tyo, eds., 8160, pp. 312 - 329, International Society for Optics and Photonics, SPIE, 2011.

[10] M. Casti, S. Fineschi, and et al., "Calibration of the liquid crystal visible-light polarimeter for the Metis/Solar Orbiter coronagraph," in Space Telescopes and Instrumentation 2018: Optical, Infrared, and Millimeter Wave, M. Lystrup, H. A. MacEwen, G. G. Fazio, N. Batalha, N. Siegler, and E. C. Tong, eds., 10698, pp. 930 - 943, International Society for Optics and Photonics, SPIE, 2018.

[11] M. Casti, S. Fineschi, and et al., "Metis/Solar Orbiter polarimetric visible light channel calibration," in International Conference on Space Optics - ICSO 2018, Z. Sodnik, N. Karafolas, and B. Cugny, eds., 11180, pp. 1255 - 1269, International Society for Optics and Photonics, SPIE, 2019.

[12] A. G. de Wijn, J. T. Burkepile, S. Tomczyk, P. G. Nelson, P. Huang, and D. Gallagher, "Stray light and polarimetry considerations for the COSMO K-Coronagraph," in Ground-based and Airborne Telescopes IV, L. M. Stepp, R. Gilmozzi, and H. J. Hall, eds., Society of Photo-Optical Instrumentation Engineers (SPIE) Conference Series 8444, p. 84443N, Sept. 2012.

[13] G. E. Brueckner, R. A. Howard, M. J. Koomen, C. M. Korendyke, D. J. Michels, J. D. Moses, D. G. Socker, K. P. Dere, P. L. Lamy, A. Llebaria, M. V. Bout, R. Schwenn, G. M. Simnett, D. K. Bedford, and C. J. Eyles, "The Large Angle Spectroscopic Coronagraph (LASCO)," Solar Phys. 162, pp. 357-402, Dec. 1995.

[14] S. Fineschi, M. Casti, G. Capobianco, G. Massone, F. Landini, M. Deffacis, and A. Bellomo, "The optical payload system facility," in 2019 IEEE 5th International Workshop on Metrology for AeroSpace (MetroAeroSpace), pp. 309-313, 2019.

[15] J. R. Lemen, A. M. Title, D. J. Akin, P. F. Boerner, C. Chou, J. F. Drake, D. W. Duncan, C. G. Edwards, F. M. Friedlaender, G. F. Heyman, N. E. Hurlburt, N. L. Katz, G. D. Kushner, M. Levay, R. W. Lindgren, D. P. Mathur, E. L. McFeaters, S. Mitchell, R. A. Rehse, C. J. Schrijver, L. A. Springer, R. A. Stern, T. D. Tarbell, J.-P. Wuelser, and et al., "The Atmospheric Imaging Assembly (AIA) on the Solar Dynamics Observatory (SDO)," Solar Phys. 275, pp. 17-40, Jan. 2012.

[16] W. D. Pesnell, B. J. Thompson, and P. C. Chamberlin, "The Solar Dynamics Observatory (SDO)," Solar Phys. 275, pp. 3-15, Jan. 2012.

[17] R. Schwenn, B. Inhester, S. P. Plunkett, A. Epple, B. Podlipnik, D. K. Bedford, C. J. Eyles, G. M. Simnett, S. J. Tappin, M. V. Bout, P. L. Lamy, A. Llebaria, G. E. Brueckner, K. P. Dere, R. A. Howard, M. J. Koomen, C. M. Korendyke, D. J. Michels, J. D. Moses, N. E. Moulton, S. E. Paswaters, D. G. Socker, O. C. St. Cyr, and D. Wang, "First View of the Extended Green-Line Emission Corona At Solar Activity Minimum Using the Lasco-C1 Coronagraph on SOHO," Solar Phys. 175, pp. 667-684, Oct. 1997.

[18] P. Lamy, A. Llebaria, B. Boclet, H. Gilardy, M. Burtin, and O. Floyd, "Coronal Photopolarimetry with the LASCO-C2 Coronagraph over 24 Years [1996 - 2019]," Solar Phys. 295, p. 89, July 2020.

[19] P. Lamy, H. Gilardy, A. Llebaria, E. Quémerais, and F. Ernandez, "LASCO-C3 Observations of the Kand F-Coronae over 24 Years (1996 - 2019): Photopolarimetry and Electron Density Distribution," Solar Phys. 296, p. 76, Apr. 2021.

[20] G. L. Withbroe, J. L. Kohl, H. Weiser, and R. H. Munro, "Probing the solar wind acceleration region using spectroscopic techniques.," Space Sci. Rev. 33, pp. 17-52, Mar. 1982.

[21] G. Noci, J. L. Kohl, and G. L. Withbroe, "Solar Wind Diagnostics from Doppler-enhanced Scattering," Ap.J. 315, p. 706, Apr. 1987.

[22] Romoli, M., Antonucci, E., Spadaro, D., Capuano, G.E., Downs, C., Fineschi, s., Landini, F., Liberatore, A., Susino, R., Uslenghi, M., Wang, Y. M., Andretta, V., Bemporad, A., Capobianco, G., Casti, M., DaDeppo, V., and et al., "First light observations of the wind in the outer solar corona with the metis coronagraph," $A \mathscr{E} A$, in press 2021. 